\title{
A retrospective study of predictive factors for effective aripiprazole augmentation of antidepressant therapy in treatment-resistant depression
}

\author{
This article was published in the following Dove Press journal: \\ Neuropsychiatric Disease and Treatment \\ 10 May 2016 \\ Number of times this article has been viewed
}

\section{Hiroko Sugawara ${ }^{1,2}$ \\ Kaoru Sakamoto' \\ Tsuyoto $\mathrm{Harada}^{3}$ \\ Satoru Shimizu ${ }^{4}$ \\ Jun Ishigooka'}

'Department of Psychiatry, Tokyo Women's Medical University, ${ }^{2}$ Support

Center for Women Health Care

Professionals and Researchers,

Tokyo Women's Medical University,

Shinjuku-ku, ${ }^{3}$ Department of

Psychiatry, Tokyo Women's Medical

University Medical Center East,

Arakawa-ku, ${ }^{4}$ Department of

Research, Medical Research Institute,

Tokyo Women's Medical University,

Shinjuku-ku, Tokyo, Japan
Correspondence: Kaoru Sakamoto

Department of Psychiatry, Tokyo

Women's Medical University, 8-I

Kawada-cho, Shinjuku-ku, Tokyo

162-8666, Japan

$\mathrm{Tel}+8 \mathrm{I} 333538$ I I I

Fax +81333518979

Email sakamoto.kaoru@twmu.ac.jp
Background: Several studies have evaluated the efficacy and tolerability of aripiprazole for augmentation of antidepressant therapy for treatment-resistant depression (TRD). Here, we investigated the efficacy of aripiprazole augmentation for TRD including both major depressive disorder and bipolar disorder and the clinical predictors of treatment efficacy in a Japanese population.

Methods: Eighty-five depressed Japanese patients who underwent aripiprazole augmentation therapy after failing to respond satisfactorily to antidepressant monotherapy were included in the study. Treatment responses were evaluated based on Clinical Global Impression Improvement scores assessed 8 weeks after initiation of aripiprazole administration. We compared demographic and diagnostic variables, psychiatric medication variables, and clinical variables between remission and nonremission groups.

Results: The aripiprazole augmentation remission rate was 36.5\%. Multiple logistic regression analysis indicated that aripiprazole augmentation was significantly more effective for bipolar depression than for major depressive disorder, and both absence of comorbid anxiety disorders and current episode duration $>3$ months were significantly associated with the efficacy of aripiprazole augmentation.

Conclusion: Polarity of depression, comorbidity of anxiety disorders, and current episode duration may predict the efficacy of aripiprazole augmentation for TRD including both major depressive disorder and bipolar disorder. Among them, comorbidity of anxiety disorders was significantly related to the efficacy for TRD including only major depressive disorder. Additional studies are needed to examine the association between the efficacy of aripiprazole augmentation and bipolarity, and these findings should be validated further in a prospective study.

Keywords: TRD, aripiprazole, predictor, bipolar depression, anxiety disorder

\section{Introduction}

Many second-generation antidepressant agents with high tolerability are widely used for standard antidepressant therapy. Approximately two-thirds of depressed patients receiving initial antidepressant therapy, however, do not achieve remission. ${ }^{1,2}$ These patients are considered to have treatment-resistant depression (TRD), although there is currently no official definition of TRD. ${ }^{1}$ The use of non-antidepressant agents to augment the effects of an antidepressant, as well as switching to or combining with another class of antidepressant agents, are promising strategies for TRD. ${ }^{3}$ Non-antidepressant agents widely used for the augmentation strategy include lithium, atypical antipsychotics 
(AAs), and thyroid hormones. The efficacy of augmentation with lithium has been evaluated by a meta-analysis of ten randomized placebo-controlled trials. ${ }^{4} \mathrm{We}$ previously investigated clinical predictors of the efficacy of lithium augmentation, focusing on the polarity of depression and bipolarity, and found that lithium augmentation is significantly more effective for depressed patients with a diagnosis changed from major depressive disorder to bipolar disorder than for those without. In addition, a family history of major depressive disorder or bipolar disorder in a first-degree relative is the most reliable predictor of lithium augmentation efficacy. ${ }^{5}$ Moreover, a long-term follow-up study by another group also indicated that bipolarity is related to a positive response to lithium augmentation in TRD and that a family history of bipolar disorder indicates lithium responders. ${ }^{6}$

Aripiprazole was the first AA approved by the United States Food and Drug Administration for use as an augmentation agent in the treatment of TRD. The efficacy and tolerability of aripiprazole augmentation have been reported in both Caucasian populations ${ }^{7,8}$ and an Asian population. ${ }^{9}$

Here, we retrospectively investigated the efficacy of aripiprazole augmentation for treatment of TRD, and evaluated the clinical predictors of treatment efficacy. Unrecognized bipolar disorder could be related to antidepressant resistance in major depressive disorder. ${ }^{10-13}$ Because previous studies demonstrated that the diagnosis of $>50 \%$ of TRD patients is subsequently changed from major depressive disorder to bipolar disorder, ${ }^{11,14}$ and a large-scale cohort study revealed an association between a history of poor response to antidepressants in major depressive disorder and a subsequent diagnosis change to bipolar disorder, ${ }^{15}$ we enrolled depressed patients with both major depressive disorder and bipolar disorder in our study.

\section{Methods}

\section{Study design}

This multicenter retrospective study was conducted from June 2006 to June 2012 at five sites in Japan: the Department of Psychiatry of the Tokyo Women's Medical University Hospital, the Department of Psychiatry of the Tokyo Women's Medical University Medical Center East, the Fuku Clinic, Murakami Hospital, and the Ms Clinic. Data were extracted from the medical records. The criterion for TRD in this study was failing to satisfactorily respond to antidepressant monotherapy, which is the administration of a maximum dose of at least one antidepressant for $>6$ weeks. This criterion corresponds to Stage I of TRD defined by Thase and Rush. ${ }^{16}$ Eighty-five depressed patients considered to have TRD who underwent aripiprazole augmentation therapy were included. Eligible patients met the Text Revision of the Diagnostic and Statistical Manual of Mental Disorders (DSM) IV (DSM-IV-TR) criteria for a major depressive episode and had an axis I diagnosis of major depressive disorder or bipolar disorder. With regard to comorbidity, patients with comorbid anxiety disorders (such as panic disorder, social anxiety disorder, generalized anxiety disorder, and obsessive compulsive disorder) were included, and patients with other comorbid psychiatric disorders, including axis II disorders, were excluded. Patients who underwent augmentation with other AAs before aripiprazole augmentation were also eligible. Aripiprazole augmentation was administered according to the clinician's judgment. Aripiprazole was added to an adequately dosed antidepressant agent, and the aripiprazole dose depended on the clinical response, including side effects. Treatment responses were evaluated based on Clinical Global Impression Improvement (CGI-I) scores assessed 8 weeks after initiating aripiprazole administration. Subjects with scores of 1 (very much improved) and 2 (much improved) were classified as the remission group, whereas those with scores ranging from 3 to 7 (very much worse) were classified as the nonremission group. We compared the two groups in terms of the demographic and diagnostic variables (eg, age, sex, diagnosis, and comorbid anxiety disorders), psychiatric medication variables (eg, class of antidepressant, final dose of aripiprazole, and premedication with other AAs), and clinical variables (eg, severity and number of recurrent major depressive episodes, duration of current major depressive episode, and lack of response to antidepressant therapy). Severity of illness was evaluated based on Clinical Global Impression Severity scores at the initiation of aripiprazole administration: subjects with scores of 3 (mildly ill) or 4 (moderately ill) were classified into the moderate group, whereas those with scores ranging from 5 (markedly ill) to 7 (among the most extremely ill) were classified into the severe group. No subjects had a score of 1 (normal, not at all ill) or 2 (borderline mentally ill). The study was conducted in accordance with the Declaration of Helsinki, and the ethics committee of the Department of Psychiatry of the Tokyo Women's Medical University Hospital, which was the principal site of this study, approved the protocol. Written informed consent from the patients was not obtained because this study was retrospective. We have informed the patients about the study using bulletin board posting, and obtained oral agreement.

\section{Statistical analyses}

A chi-square test or Fisher's exact test was conducted to compare categorical variables between the two groups. Multiple logistic regression analysis was performed between the two 
groups as a dependent variable selected the independent variables for the model using the stepwise method based on Akaike's information criterion. ${ }^{17}$ All statistical analyses were conducted using JMP statistical software (Version 11; SAS Institute Inc., Cary, NC, USA). Each test was conducted at a significance level of $P<0.05$.

\section{Results}

Among the 85 depressed patients, 31 were classified into the remission group and 54 into the nonremission group (Table 1); that is, the efficacy rate of aripiprazole augmentation was $36.5 \%$ (31/85). Age and sex did not significantly differ between the two groups. Fourteen patients were diagnosed with bipolar disorder and underwent aripiprazole augmentation for the depressive state, and the rate of bipolar disorder in the remission group was significantly higher $(P=0.031)$ (Table 1). On the other hand, the rate of comorbid anxiety disorders in the remission group was significantly lower $(P=0.026)$ (Table 1$)$. None of the psychiatric medication variables were related to the efficacy of aripiprazole augmentation. Patients with a current episode duration $<3$ months were less likely to respond to aripiprazole augmentation than the patients with a longer-duration current episode $(P=0.085)$ (Table 1). In multiple logistic regression analysis, diagnosis, comorbid anxiety disorders, and duration of current episode were extracted as independent variables among all the variables using Akaike's information criterion with a stepwise method. Aripiprazole augmentation was significantly more effective for bipolar depression (odds ratio $=3.64,95 \%$ confidence interval $=1.05-14.29, P=0.041$ ) (Table 2) than for major depressive disorder, and both absence of comorbid anxiety disorders and current episode duration $>3$ months were significantly associated with the efficacy of aripiprazole augmentation (odds ratio $=8.87,95 \%$ confidence interval $=1.54-169.44, P=0.011$ and odds ratio $=6.76,95 \%$ confidence interval $=1.10-131.79, P=0.037$, respectively) (Table 2). Furthermore, even if the subjects were limited to those with major depressive disorder, only the absence of comorbid anxiety disorders was significantly associated with the efficacy of aripiprazole augmentation (odds ratio $=6.61$, $95 \%$ confidence interval $=1.15-125.67, P=0.032$ ).

\section{Discussion}

In this multicenter retrospective study including Japanese subjects with major depressive disorder and bipolar disorder, the remission rate of aripiprazole augmentation was $36.5 \%$. This rate is slightly higher than that reported in a multicenter, randomized, placebo-controlled study in an Asian population, in which the remission rates were $30.4 \%$ for a flexible dose of $3-15 \mathrm{mg}$ and $32.5 \%$ for a fixed dose of $3 \mathrm{mg} .{ }^{9}$ The remission rate limited to major depressive disorder patients, however, was $31.0 \%(22 / 71)$. This rate is similar to that of a previous study. ${ }^{9}$ Other studies reported that aripiprazole augmentation is effective for mildly, moderately, and severely ill patients, ${ }^{18,19}$ and we also found that efficacy was unrelated to illness severity. Our finding that other variables, such as age, sex, class of antidepressant, number of recurrent major depressive episodes, and lack of response to antidepressant therapy, were unrelated to the efficacy of aripiprazole augmentation is also consistent with the results of a previous study. ${ }^{18}$ Although about half of the subjects in our study were receiving $>3 \mathrm{mg}$ of aripiprazole

Table I Demographic and clinical characteristics of subjects

\begin{tabular}{|c|c|c|c|}
\hline Characteristics & Remission group $(n=31)$ & Nonremission group $(n=54)$ & $P$-values \\
\hline \multicolumn{4}{|l|}{ Demographic and diagnostic variables } \\
\hline Age (20-30 years $/ 40-50$ years $/ 60-70$ years) & $6 / 16 / 9(19.4 \% / 51.6 \% / 29.0 \%)$ & I5/25/I 4 (27.8\%/46.3\%/25.9\%) & $P=0.687$ \\
\hline Sex (men/women) & $15 / 16(48.4 \% / 51.6 \%)$ & $23 / 31(42.6 \% / 57.4 \%)$ & $P=0.60 \mathrm{I}$ \\
\hline Diagnosis (MD/BD) & $22 / 9$ & $49 / 5$ & $P=0.03 I^{*}$ \\
\hline Comorbid anxiety disorders, n (\%) & I (3.2\%) & $12(22.2 \%)$ & $P=0.026^{*}$ \\
\hline \multicolumn{4}{|l|}{ Psychiatric medication variables } \\
\hline Class of antidepressant (SSRI or SNRI/TCA/both) & $|7 / 3 /| 1$ & $34 / 4 / 16$ & $P=0.759$ \\
\hline Final dose of aripiprazole, $>3 \mathrm{mg}, \mathrm{n}(\%)$ & $13(41.9 \%)$ & $31(57.4 \%)$ & $P=0.169$ \\
\hline Premedication of other AAs, $n(\%)$ & $10(32.3 \%)$ & $18(33.3 \%)$ & $P=0.919$ \\
\hline \multicolumn{4}{|l|}{ Clinical variables } \\
\hline Severity (moderate group/severe group) & $24 / 7$ & $42 / 12$ & $P=1.000$ \\
\hline Number of recurrent major depressive episodes, $>3, \mathrm{n}(\%)$ & $10(32.3 \%)$ & $13(24.1 \%)$ & $P=0.454$ \\
\hline Lack of response to antidepressant therapy, $>2, n(\%)$ & $18(58.1 \%)$ & $24(44.4 \%)$ & $P=0.227$ \\
\hline Duration of current episode, $<3$ months, n (\%) & I (3.2\%) & $9(16.07 \%)$ & $P=0.085$ \\
\hline
\end{tabular}

Note: $* P<0.05$.

Abbreviations: AAs, atypical antipsychotics; BD, bipolar disorder; MD, major depressive disorder; SNRI, serotonin-noradrenalin reuptake inhibitor; SSRI, selective serotonin reuptake inhibitor; TCA, tricyclic antidepressant. 
Table 2 Predictors of efficacy of aripiprazole augmentation

\begin{tabular}{llll}
\hline Predictor & OR $^{\mathrm{a}}$ & $\mathbf{9 5 \%} \mathbf{C l}$ & $\boldsymbol{P}$-value \\
\hline Bipolar depression & 3.64 & $\mathrm{I} .05-14.29$ & $P=0.04 \mathrm{I}^{*}$ \\
Absence of comorbid anxiety disorders & 8.87 & $\mathrm{I} .54-169.44$ & $P=0.0 \mathrm{II}$ \\
Duration of current episode $>3$ months & 6.76 & $\mathrm{I} .10-13 \mathrm{I} .79$ & $P=0.037^{*}$
\end{tabular}

Notes: $* P<0.05$. ${ }^{~}$ Calculated by multiple logistic regression analysis using independent variables selected by the stepwise method based on Akaike's information criterion. ${ }^{17}$

Abbreviations: $\mathrm{Cl}$, confidence interval; OR, odds ratio.

as the final dose, the final dose was not related to efficacy. Aripiprazole augmentation at both a flexible dose and a fixed dose was found to be effective for TRD patients in Japan, ${ }^{9}$ suggesting that the aripiprazole dose should be selected on the basis of clinical effect and tolerability. The results of a meta-analysis and a nationwide population-based study support antidepressant augmentation with AAs for TRD. ${ }^{20,21}$ In our study, more than half of the subjects had taken other AAs before taking aripiprazole, and the presence or absence of premedication with other AAs was not related to the efficacy of aripiprazole augmentation. Recent studies of a network meta-analysis showed that aripiprazole was the most robust evidence-based option among various augmentation agents, including other AAs, for augmentation therapy in TRD patients. $^{22,23}$ If augmentation of antidepressants with other AAs fails to achieve remission, aripiprazole augmentation is worth trying.

Our findings indicate that aripiprazole augmentation may be more effective for bipolar depression than for major depressive disorder, and that both absence of comorbid anxiety disorders and duration of current episode of $>3$ months may predict the efficacy of aripiprazole augmentation. Mood disorders, including major depressive disorder, bipolar disorder, and bipolar spectrum disorder, are commonly comorbid with anxiety disorders, ${ }^{24}$ and a large number of individuals with bipolar disorder and bipolar spectrum disorder will meet the diagnostic criteria for an anxiety disorder during their lifetime. ${ }^{25}$ In the present study, the rate of comorbid anxiety disorders in patients with major depressive disorder was higher $(12 / 71 ; 16.9 \%)$ than that in patients with bipolar disorder $(1 / 14 ; 7.1 \%)$. Based on the results of the multiple logistic regression analysis for subjects with major depressive disorder and bipolar disorder and for those with only major depressive disorder, comorbid anxiety disorder was considered to be a negative predictor of aripiprazole augmentation. Previous studies reported that anxiety disorder comorbidity with mood disorder increases depression severity and suicide risk. ${ }^{26-29}$ Our findings suggest that anxiety disorders comorbid with both major depressive disorder and bipolar disorder may increase resistance to treatment. Obsessive-compulsive disorder (OCD) was included in anxiety disorders in the present study based on DSM-IV-TR as well as previous reports, ${ }^{25-29}$ however, the category of OCD has been separated from anxiety disorders in DSM-5, and future studies are needed to evaluate the comorbidity of anxiety disorders excluding OCD in mood disorders.

In a previous study, we found that lithium augmentation is more effective for depressed patients with a diagnosis change from major depressive disorder to bipolar disorder than for those without, and that a family history of major depressive disorder or bipolar disorder in a first-degree relative is the most reliable predictor of the efficacy of lithium augmentation. $^{5}$ In the present study, aripiprazole augmentation was also more effective for patients with bipolar disorder than for patients with major depressive disorder, but the association between the efficacy of aripiprazole augmentation and family history of mood disorders could not be investigated because information about the family history of the patients in the present study was not available. The association between the efficacy of aripiprazole augmentation and a brief major depressive episode ( $<3$ months) disappeared when the subjects were limited to patients with major depressive disorder $(P=0.105)$. A brief major depressive episode ( $<3$ months $)$ is reported to be a criterion for bipolar spectrum disorder, ${ }^{30}$ and the association between the efficacy of aripiprazole augmentation and other factors of bipolarity requires further prospective studies comprising a larger number of subjects.

\section{Limitations}

There are some limitations to the present study. The main limitation is that the study was retrospectively designed, and the sample size was small. Assessment of the efficacy and severity were based on CGI-I and Clinical Global Impression Severity scores, and an accurate evaluation was not performed using a rating scale, such as the Hamilton Depression Rating Scale. In addition, due to the retrospective nature of our study, important clinical information might have been overlooked. Senior psychiatrists follow-up all patients, and therefore, the information described in their medical records is considered accurate, which may minimize these limitations.

\section{Conclusion}

Our findings suggest that the polarity of depression, comorbidity of anxiety disorders, and current episode duration may predict the efficacy of aripiprazole augmentation for TRD including both major depressive disorder and bipolar disorder. Among them, comorbidity of anxiety disorders was 
significantly related to the efficacy for TRD including only major depressive disorder. Additional studies are needed to examine the association between the efficacy of aripiprazole augmentation and bipolarity, and our results should be validated by a prospective study.

\section{Acknowledgments}

The authors would like to thank T Fukunaga, MD (Fuku Clinic), K Murakami, MD (Murakami Hospital), and M Sakurada, MD (Ms Mental Clinic) for their assistance with data collection. This study was supported by Toshi Miyahara research grant for Support Center for Women Health Care Professionals and Researchers, Tokyo Women's Medical University.

\section{Author contributions}

All authors contributed to the conception and design of the study. HS, TH, and KS contributed to data collection. HS performed the statistical analyses and wrote the manuscript. SS provided advice regarding the statistical analysis methods. All authors contributed toward data analysis, drafting and revising the paper and agree to be accountable for all aspects of the work.

\section{Disclosure}

During the past 3 years, TH has received speakers' bureau honoraria from GlaxoSmithKline, Otsuka Pharmaceuticals, Meiji Seika Pharma, and Tanabe Mitsubishi. KS has received speakers' bureau honoraria from Eli Lilly, GlaxoSmithKline, Otsuka Pharmaceuticals, Meiji Seika Pharma, and Pfizer Pharmaceuticals. JI has received speakers' bureau honoraria from Dainihon Sumitomo, Mochida, Shionogi, and Otsuka Pharmaceuticals. The authors report no other conflicts of interest in this work.

\section{References}

1. Fava M. Diagnosis and definition of treatment-resistant depression. Biol Psychiatry. 2003;53(8):649-659.

2. Rush AJ, Trivedi MH, Wisniewski SR, et al. Acute and longerterm outcomes in depressed outpatients requiring one or several treatment steps: a STAR*D report. Am J Psychiatry. 2006;163(11): 1905-1917.

3. Sackeim HA. The definition and meaning of treatment-resistant depression. J Clin Psychiatry. 2001;62(Suppl 16):10-17.

4. Crossley NA, Bauer M. Acceleration and augmentation of antidepressants with lithium for depressive disorders: two meta-analyses of randomized, placebo-controlled trials. J Clin Psychiatry. 2007;68(6):935-940.

5. Sugawara H, Sakamoto K, Harada T, Ishigooka J. Predictors of efficacy in lithium augmentation for treatment-resistant depression. J Affect Disord. 2010;125(1-3):165-168.

6. Inoue $\mathrm{T}$, Abekawa $\mathrm{T}$, Nakagawa $\mathrm{S}$, et al. Long-term naturalistic follow-up of lithium augmentation: relevance to bipolarity. J Affect Disord. 2011; 129(1-3):64-67.
7. Berman RM, Marcus RN, Swanink R, et al. The efficacy and safety of aripiprazole as adjunctive therapy in major depressive disorder: a multicenter, randomized, double-blind, placebo-controlled study. J Clin Psychiatry. 2007;68(6):843-853.

8. Marcus RN, McQuade RD, Carson WH, et al. The efficacy and safety of aripiprazole as adjunctive therapy in major depressive disorder: a second multicenter, randomized, double-blind, placebo-controlled study. J Clin Psychopharmacol. 2008;28(2):156-165.

9. Kamijima K, Higuchi T, Ishigooka J, et al. Aripiprazole augmentation to antidepressant therapy in Japanese patients with major depressive disorder: a randomized, double-blind, placebo-controlled study (ADMIRE study). J Affect Disord. 2013;151(3):899-905.

10. Akiskal HS, Mallya G. Criteria for the "soft" bipolar spectrum: treatment implications. Psychopharmacol Bull. 1987;23(1):68-73.

11. Correa R, Akiskal H, Gilmer W, Nierenberg AA, Trivedi M, Zisook S. Is unrecognized bipolar disorder a frequent contributor to apparent treatment resistant depression? J Affect Disord. 2010;127(1-3):10-18.

12. Inoue $T$, Nakagawa $S$, Kitaichi $Y$, et al. Long-term outcome of antidepressant-refractory depression: the relevance of unrecognized bipolarity. J Affect Disord. 2006;95(1-3):61-67.

13. Kornstein SG, Schneider RK. Clinical features of treatment-resistant depression. J Clin Psychiatry. 2001;62(Suppl 16):18-25.

14. Sharma V, Khan M, Smith A. A closer look at treatment resistant depression: is it due to a bipolar diathesis? J Affect Disord. 2005; 84(2-3):251-257.

15. Li CT, Bai YM, Huang YL, et al. Association between antidepressant resistance in unipolar depression and subsequent bipolar disorder: cohort study. Br J Psychiatry. 2012;200(1):45-51.

16. Thase ME, Rush AJ. When at first you don't succeed: sequential strategies for antidepressant nonresponders. J Clin Psychiatry. 1997;58 Suppl 13:23-29.

17. Yamaoka K, Nakagawa T, Uno T. Application of Akaike's information criterion (AIC) in the evaluation of linear pharmacokinetic equations. J Pharmacokinet Biopharm. 1978;6(2):165-175.

18. Ozaki N, Otsubo T, Kato M, Higuchi T, Ono H, Kamijima K; ADMIRE Study Group. Efficacy of aripiprazole augmentation in Japanese patients with major depressive disorder: A subgroup analysis and MontgomeryAsberg Depression Rating Scale and Hamilton Rating Scale for Depression item analyses of the Aripiprazole Depression Multicenter Efficacy study. Psychiatry Clin Neurosci. 2015;69(1):34-42.

19. Stewart TD, Hatch A, Largay K, et al. Effect of symptom severity on efficacy and safety of aripiprazole adjunctive to antidepressant monotherapy in major depressive disorder: A pooled analysis. J Affect Disord. 2014;162:20-25.

20. Lin CY, Tsai GE, Wang HS, et al. Effectiveness of aripiprazole, olanzapine, quetiapine, and risperidone augmentation treatment for major depressive disorder: a nationwide population-based study. J Clin Psychiatry. 2014;75(9):e924-e931.

21. Papakostas GI, Shelton RC, Smith J, Fava M. Augmentation of antidepressants with atypical antipsychotic medications for treatmentresistant major depressive disorder: a meta-analysis. J Clin Psychiatry. 2007;68(6):826-831.

22. Zhou X, Ravindran AV, Qin B, et al. Comparative efficacy, acceptability, and tolerability of augmentation agents in treatment-resistant depression: systematic review and network meta-analysis. $J$ Clin Psychiatry. 2015;76(4):e487-e498.

23. Zhou X, Keitner G, Qin B, et al. Atypical antipsychotic augmentation for treatment-resistant depression: a systematic review and network meta-analysis. Int J Neuropsychopharmacol. 2015;18(11):pyv060.

24. Goes FS, McCusker MG, Bienvenu OJ, et al; National Institute of Mental Health Genetics Initiative Bipolar Disorder Consortium. Comorbid anxiety disorders in bipolar disorder and major depression: familial aggregation and clinical characteristics of co-morbid panic disorder, social phobia, specific phobia and obsessive-compulsive disorder. Psychol Med. 2012;42(7):1449-1459.

25. Merikangas KR, Akiskal HS, Angst J, et al. Lifetime and 12-month prevalence of bipolar spectrum disorder in the National Comorbidity Survey replication. Arch Gen Psychiatry. 2007;64(5):543-552. 
26. Gili M, Garcia Toro M, Armengol S, Garcia-Campayo J, Castro A, Roca M. Functional impairment in patients with major depressive disorder and comorbid anxiety disorder. Can J Psychiatry. 2013;58(12):679-686.

27. Lee JH, Dunner DL. The effect of anxiety disorder comorbidity on treatment resistant bipolar disorders. Depress Anxiety. 2008;25(2): 91-97.

28. Thaipisuttikul $P$, Ittasakul $P$, Waleeprakhon $P$, Wisajun $P$, Jullagate $S$. Psychiatric comorbidities in patients with major depressive disorder. Neuropsychiatr Dis Treat. 2014;10:2097-2103.
29. Vieta E, Colom F, Martinez-Aran A, Benabarre A, Reinares M, Gasto C. Bipolar II disorder and comorbidity. Compr Psychiatry. 2000;41(5): 339-343.

30. Ghaemi SN, Hsu DJ, Ko JY, Baldassano CF, Kontos NJ, Goodwin FK. Bipolar spectrum disorder: a pilot study. Psychopathology. 2004;37(5): $222-226$.

\section{Publish your work in this journal}

Neuropsychiatric Disease and Treatment is an international, peerreviewed journal of clinical therapeutics and pharmacology focusing on concise rapid reporting of clinical or pre-clinical studies on a range of neuropsychiatric and neurological disorders. This journal is indexed on PubMed Central, the 'PsycINFO' database and CAS, and is the official journal of The International Neuropsychiatric Association (INA). The manuscript management system is completely online and includes a very quick and fair peer-review system, which is all easy to use. Visit http://www.dovepress.com/testimonials.php to read real quotes from published authors.

Submit your manuscript here: http://www.dovepress.com/neuropsychiatric-disease-and-treatment-journal 